\title{
SPATIAL LOCALIZED DOUBLE-QUANTUM NMR HETERONUCLEAR COHERENCE TRANSFER IN SOLIDS BY INDIRECT DETECTION
}

\author{
D.E. Demco and I. Ardelean \\ Technical University, Department of Physics, 3400 Cluj-Napoca, Romania
}

(Received February 7, 1996; in final form April 24, 1996)

The spatial localization of the double-quantum NMR heteronuclear coherence transfer in solids, in the presence of a main magnetic field gradient is analyzed. The indirect detection procedure by which the dipolar order of the abundant spin system with $I=1 / 2$ is transferred to the double-quantum coherence of the quadrupole nucleus with $S=1$ and back to the $I$-spin dipolar order is considered. The slice : rofile and the cross-relaxation rates are evaluated as a function of the experimental parameters and the sample relevant NMR quantities. The possibility to use this slice selective procedure for imaging and volume localized spectroscopy of quadrupole nuclei in high magnetic fields is discussed.

PACS numbers: 76.70.-r, 76.70.Fz, 33.35.-q

\section{Introduction}

During the last few years a considerable progress in the field of spatially resolved NMR spectroscopy and NMR imaging in solid materials [1-3] (and references therein) has been made. Since the spatial resolution is ultimately limited by sensitivity, most experiments have been performed on protons so far. However, the proton spectra in solids are broadened by strong homonuclear dipolar intersections, the influence of which must be suppressed to achieve an efficient spatial encoding. The problem of severe homogeneous line broadening can be circumvented by imaging of magnetically dilute nuclei, e.g., ${ }^{13} \mathrm{C},{ }^{2} \mathrm{H}$, etc. [4-8]. The heterogeneously broadened lines of the dilute nuclci with the spin $S=1 / 2$, like ${ }^{13} \mathrm{C}$, can be more easily narrowed by high-power proton decoupling combined in some cases with magic-angle sample spinning. For the quadrupole nuclei the total multiple quantum NMR coherence is insensitive to the first order quadrupole couplings which leads to the possibility to record high-resolution NMR spectra [9-13]. Indirect heteronuclear coherence transfer via dipolar order allows the observation of ${ }^{14} \mathrm{~N}$ double-quantum (DQ) transitions for quadrupolar splitting up to 
$2 \mathrm{MHz}$ [11]. The versatility of this technique is superior to that of direct spin-lock cross-polarization. Moreover, these approaches give access to the rich structural and dynamical information of ${ }^{13} \mathrm{C},{ }^{15} \mathrm{~N}$, etc, and quadrupole nuclei (e.g. ${ }^{2} \mathrm{H}$ or ${ }^{14} \mathrm{~N}$ ) spectra which is the most important prerequisite for useful application of localized NMR in solids.

In the field of solid-state imaging or volume-selective spectroscopy and efficient method for slice-selection is required in order to reduce the duration of the experiments. The first method used for slice-selection in imaging [14] as well as for volume-selection in solids is the LOSY technique $[15,16]$, which is based on the off-resonance sensitivity of the spin-locking procedure. Another methods applied in the case of protons are based on DANTE sequence [17] and Lee-Goldburg line-narrowing technique $[18,19]$.

A slice-selection method for localized ${ }^{13} \mathrm{C}$ spectroscopy for solids and liquids was recently proposed $[20,21,1]$ and the efficiency of the technique was analyzed theoretically and experimentally [22-26]. The sequence is based on single-quantum (SQ) cross-polarization from abundant to dilute spins in the presence of a main magnetic field gradient. The efficiency of the cross-polarization is strongly sensitive to resonance offsets and consequently shows a strong spatial dependence in the presence of field gradients. The sensitivity enhancement of the rare spins and of localized excitation is achieved at the same time. Following this process, the full spectroscopic information of the dilute spins may be exploited using suitable pulse sequences.

The main goal of this paper is to analyze the slice profile produced in the heteronuclear coherence transfer by double-quantum transitions in the presence of the main magnetic field. We shall concentrate here on the excitation and detection of double-quantum coherence by cross-polarization via dipolar order. This technique is suitable for quadrupole nuclei with large quadrupolar splitting and can be applied to record double-quantum high-resolution spectra by observing proton resonance.

The paper is organized as follows. In Sec. 2 we introduce the space localized double-quantum Hamiltonian which describes the process of coherence transfer by double-quantum transitions for an abundant spin system $I=1 / 2$ and a dilute spin system $S=1$, with quadrupolar interaction in the presence of a field gradient. The spatial localized spin dynamics is discussed in Sec. 3. The slice profile obtained by indirect double-quantum detection via cycled dipolar order transfer are evaluated in Sec. 4. The possibility of volume-selective investigations of the structure and dynamics of quadrupole nuclei in materials is finally discussed.

\section{Spatial localized double-quantum Hamiltonian}

In this section we recall some details related to the effective double-quantum Hamiltonian which describes double-quantum coherence transfer experiments in solids. The high field DQ-Hamiltonian in the laboratory reference frame for the experimental configuration presented in Fig. 1 is

$$
H=H_{I}+H_{S}+H_{I S}+H_{\mathrm{rf}}(t) \text {. }
$$



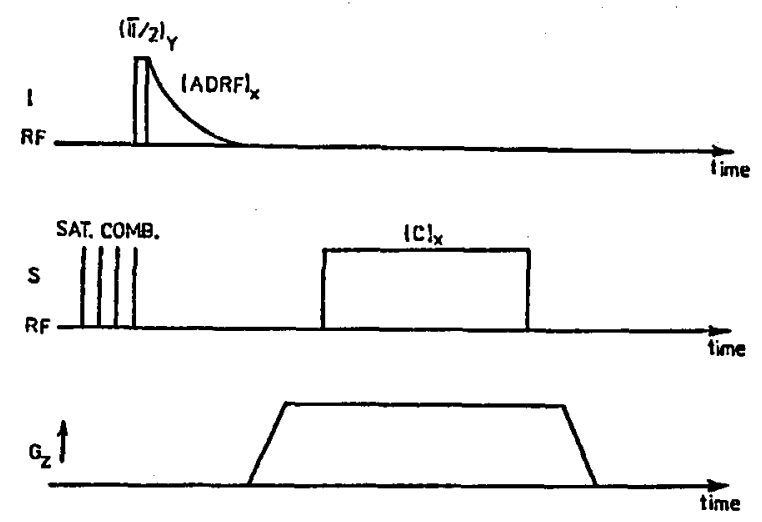

Fig. 1. The pulse sequence used for double-quantum coherence transfer for $I=1 / 2$ and $S=1$ spins performed in the presence of a main magnetic field gradient. For the ADRF procedure the field gradient is applied only during contact pulse. The quadrupolar $S$-spins are saturated before the coherence transfer process.

The Hamiltonian for the abundant $I$-spin system is

$$
H_{I}=H_{Z I}+H_{I I}^{(0)} \text {, }
$$

where the Zeeman IIamiltonian, which includes also the chemical shift, has the form

$$
H_{\mathrm{ZI}}=-\omega_{0 I} I_{z}-\sum_{z-\text { slice }} \Delta \Omega_{I}(z) I_{z}(z) .
$$

Here $\omega_{0 I}=\gamma_{I} B_{0}$ is the Larmor frequency and $\Delta \Omega_{I}(z)=\gamma_{I} G_{z} z$ with $\gamma_{I}$ being the magnetogyric ratio, $G_{z}$ is the field gradient and $z$ is the space coordinate.

The truncated homonuclear dipolar Hamiltonian is

$$
H_{I I}^{(0)}=\sum_{i<j} b_{i j}\left(I_{i} \cdot I_{j}-3 I_{i z} I_{j z}\right)
$$

with the dipolar interaction factor

$$
b_{i j}=\left(\mu_{0} / 4 \pi\right) \gamma_{I}^{2} \hbar r_{i j}^{-3} P_{2}\left(\cos \theta_{i j}\right) \text {. }
$$

The IIamiltonian $H_{S}$, which characterizes the dilute quadrupole spin-system, is

$$
H_{S}=H_{\mathrm{ZS}}+H_{\mathrm{QS}} .
$$

The Zeeman Hamiltonian $H_{\mathrm{ZS}}$, which includes also the chemical shift, has the form

$$
H_{\mathrm{Z} S}=-\omega_{0 S} S_{z}-\sum_{z-\text { slice }} \Delta \Omega_{S}(z) S_{z}(z),
$$

where $\omega_{0 S}=\gamma_{S} B_{0}$ and $\Delta \Omega_{S}(z)=\gamma_{S} G_{z} z$. The magnetogyric ratio of spin $S$ is $\gamma_{S}$. In the presence of a strong static magnetic field the truncated quadrupolar interaction can be written [10] as

$$
H_{\mathrm{QS}}=\frac{1}{3} \omega_{\mathrm{Q}}\left[3 S_{z}^{2}-S(S+1)\right]
$$


where the first order quadrupolar splitting $2 \omega_{Q}$ is given by [10]

$$
2 \omega_{Q}=\frac{3 e^{2} q Q}{4 \hbar}\left[\frac{1}{2}\left(3 \cos ^{2} \beta-1\right)+\eta \sin ^{2} \beta \cos 2 \gamma\right] \text {. }
$$

The Euler angles $(\alpha, \beta, \gamma)$ specify the orientation of quadrupole interaction with respect to the magnetic field $B_{0}$ and the asymmetry factor $\eta=\left(V_{y y}-\right.$ $\left.V_{x x}\right) / V_{z z}$ describes the deviation from the axial symmetry.

For the diluted $S$-spins in Eq. (6) we can neglect the dipolar coupling between themselves.

The Hamiltonian $H_{I S}$ describes the interaction between $I$ - and $S$-spin systems. The main contribution is given by the heteronuclear dipolar interaction

$$
H_{I S}^{(0)}=\sum_{i, m} b_{i m} I_{i z} S_{m z}
$$

with the interaction factor

$$
b_{i m}=-\left(\mu_{0} / 4 \pi\right) 2 \gamma_{I} \gamma_{S} \hbar r_{i m}^{-3} P_{2}\left(\cos \theta_{i m}\right) \text {. }
$$

In the above expressions the indices $i, j$ and $m$ refer to the $I$ and $S$ spins, respectively, $r_{i j}$ is the distance between $i$ and $j$ spins and $\theta_{i j}$ is the angle between the vector $\boldsymbol{r}_{i j}$ connecting $i$ and $j$ spins and applied magnetic field $\boldsymbol{B}_{0}$.

The interaction of the spin system with the radiofrequency magnetic fields of strengths $B_{1 I}, B_{1 S}$ and frequencies $\omega_{1 I}, \omega_{1 S}$ has the form

$$
H_{\mathrm{rf}}(t)=-2 \omega_{1 I} I_{x} \cos \omega_{I} t-2 \omega_{1 S} S_{x} \cos \omega_{S} t
$$

where $\omega_{1 I}=\gamma_{I} B_{1 I}$ and $\omega_{1 S}=\gamma_{S} B_{1 S}$.

The total Hamiltonian can be written [16] in a good approximation as a sum of $z$-slice Hamiltonians, i.e.

$$
H=\sum_{z-\text { slice }} H(z)
$$

and consequently the total density operator of the spin system has the form

$$
\rho(t)=\sum_{z-\text { slice }} \rho(z, t) \text {. }
$$

The local Liouville-von Neuman equation in the laboratory reference frame . is

$$
\mathrm{i} \frac{\partial \rho(z, t)}{\partial t}=\widehat{H}(z) \rho(z, t),
$$

where we denoted a Liouville operator corresponding to an operator $O$ by $\hat{O}$ and $\hbar=1$.

In the following we will refer only to a particular $z$-slice but for simplicity we shall drop sometimes in the following equations the explicit dependence on spatial coordinate $z$.

In the spatially localized rotating $(R)$ frame the Liouville-von Neuman equation (15) becomes

$$
\mathrm{i} \frac{\partial \rho_{R}}{\partial t}=\widehat{H}_{R} \rho_{R}
$$


where

$$
\rho_{R}=\widehat{R} \rho
$$

with

$$
R=\exp \left(-\mathrm{i} \omega_{I} I_{\underline{z}} t\right) \exp \left(-\mathrm{i} \omega_{S} S_{\underline{z}} t\right) .
$$

The total local Hamiltonian has the form

$$
H_{R}=H_{I, R}+H_{S, R}+H_{I S, R}+H_{\mathrm{rf}, R},
$$

where

$$
\begin{aligned}
& H_{I, R}=-\left(\Delta \omega_{I}+\Delta \Omega_{I}\right) I_{\underline{z}}+H_{I I}^{(0)} \\
& H_{S, R}=-\left(\Delta \omega_{S}+\Delta \Omega_{S}\right) S_{\underline{z}}+H_{\mathrm{QS}} \\
& H_{I S, R}=H_{I S}^{(0)} \\
& H_{\mathrm{rf}, R}=-\omega_{1 I} I_{\underline{x}}-\omega_{1 S} S_{\underline{x}}
\end{aligned}
$$

with $\Delta \omega_{I}=\omega_{0 I}-\omega_{I}$ and $\Delta \omega_{S}=\omega_{0 S}-\omega_{S}$. We have considered here that RF-fields are polarized along the $\underline{x}$-direction in the rotating frames $(\underline{x}, \underline{y}, \underline{z}$ are the notations for the coordinate axes in the rotating frames).

In the case of multilevel spin system it is convenient to express the Hamiltonian in terms of fictitious cartesian single-transition operators. Using these operators

$$
\begin{aligned}
& S_{x}=\sqrt{2}\left(S_{x}^{(1-2)}+S_{x}^{(2-3)}\right), \quad S_{y}=\sqrt{2}\left(S_{y}^{(1-2)}+S_{y}^{(2-3)}\right), \\
& S_{x}=2 S_{z}^{(1-3)}=2\left(S_{z}^{(1-2)}+S_{z}^{(2-3)}\right),
\end{aligned}
$$

Eqs. (20b-d) can be rewritten as

$$
\begin{aligned}
& H_{S, R}=-2\left(\Delta \omega_{S}+\Delta \Omega_{S}\right) S_{\underline{z}}^{(1-2)}+\frac{2}{3} \omega_{Q}\left(S_{\underline{z}}^{(1-2)}-S_{\underline{z}}^{(2-3)}\right), \\
& H_{I S, R}=\sum_{i, m} b_{i m} I_{\underline{i} \underline{z}}\left(2 S_{m \underline{z}}^{(1-3)}\right) \\
& H_{\mathrm{rf}, R}=-\omega_{1 I} I_{\underline{x}}-\sqrt{2} \omega_{1 S}\left(S_{\underline{x}}^{(1-2)}+S_{\underline{x}}^{(2-3)}\right) .
\end{aligned}
$$

We introduced now a tilted rotating $(T R)$ frame defined by

$$
\rho_{T R}=\widehat{T} \rho_{R}
$$

and

$$
H_{T R}=\widehat{T} H_{R} .
$$

The canonic transformation is

$$
\widehat{T}=\exp \left(\mathrm{i} \theta_{I} \widehat{I}_{\bar{y}}\right),
$$

where

$$
\tan \theta_{I}=\frac{\omega_{1 I}}{\Delta \omega_{I}+\Delta \Omega_{I}}
$$

Using the above transformations the total spin Hamiltonian takes the form 


$$
\begin{aligned}
H_{T R} & =-\omega_{\mathrm{eff}, I} I_{\bar{z}}+P_{2}\left(\cos \theta_{I}\right) H_{I I}^{(0)}+\cos \theta_{I} \sum_{i, m} b_{i m} I_{i \bar{z}} 2 S_{m \underline{z}}^{(1-3)} \\
& -\sin \theta_{I} \sum_{i, m} b_{i m} I_{i \bar{x}} 2 S_{m \underline{z}}^{(1-3)}-2\left(\Delta \omega_{S}+\Delta \Omega_{S}\right) S_{\underline{z}}^{(1-3)} \\
& -\sqrt{2} \omega_{1 S}\left[S_{\underline{x}}^{(1-2)}+S_{\underline{x}}^{(2-3)}+\frac{2}{3} \omega_{Q}\left[S_{\underline{z}}^{(1-3)}-S_{\underline{z}}^{(2-3)}\right]\right.
\end{aligned}
$$

where $\omega_{\text {eff, } I}=\left[\left(\Delta \omega_{I}+\gamma_{I} G_{z} z\right)^{2}+\omega_{1 I}^{2}\right]^{1 / 2}$. The $I$-spin tilted rotating frame has $(\bar{x}, \bar{y}, \bar{z})$-axis.

The above Hamiltonian has been written in the approximation of high-effective field, i.e. $\omega_{\mathrm{eff}, I} \gg \omega_{\mathrm{L} I}^{\prime}$, where $\omega_{\mathrm{L} I}^{\prime}$ is the local field in the tilted rotating frame. In this quantum-mechanical representation the total Hamiltonian is quasi-diagonal in the $I$-spin Liouville subspace.

To diagonalize the Hamiltonian, Eq. (26), also in the $S$-spin Liouville subspace, we make the following transformations:

$$
H_{\mathrm{DQ}}=\exp \left(-\mathrm{i} \varphi \widehat{S}_{\underline{y}}^{(1-3)}\right) \exp \left(-\mathrm{i} \frac{\pi}{2} \widehat{S}_{\underline{y}}^{(1-3)}\right) \exp \left(-\mathrm{i} \theta \widehat{S}_{\underline{y}}^{(1-2)}\right) \exp \left(\mathrm{i} \frac{\pi}{2} \widehat{S}_{\underline{y}}^{(1-3)}\right) H_{T R},
$$

where

$$
\tan \theta=\frac{2 \omega_{1 S}}{\omega_{Q}}
$$

and

$$
\tan \varphi=\frac{\omega_{e}-\omega_{Q}}{4 \Delta \Omega_{S} \cos (\theta / 2)}
$$

with $\omega_{e}=\left[\left(2 \omega_{1 S}\right)^{2}+\omega_{Q}^{2}\right]^{1 / 2}$. We have considered in Eq. $(28 \mathrm{~b})$ that $\Delta \omega_{S}=0$.

Using the transformation of Cartesian single transition operators under rotations $[27,28]$ and the approximation of weak $S$-RF field, i.e. $\omega_{1 S} \ll \omega_{Q}(\theta \ll 1)$, from Eqs. (26) and (27) we can obtain the following expression for double-quantum Hamiltonian:

$$
\begin{aligned}
H_{\mathrm{DQ}} & =-\omega_{\mathrm{eff}, I} I_{\bar{z}}+P_{2}\left(\cos \theta_{I}\right) H_{I I}^{(0)} \\
& +2 \cos \theta_{I} \cos (\theta / 2) \cos \varphi \sum_{i, m} b_{i m} I_{i \bar{z}} S_{m \underline{z}}^{(1-3)} \\
& +2 \cos \theta_{I} \cos (\theta / 2) \sin \varphi \sum_{i, m} b_{i m} I_{i \bar{z}} S_{m \underline{x}}^{(1-3)} \\
& -2 \sin \theta_{I} \cos (0 / 2) \cos \varphi \sum_{i, m} b_{i m} I_{i \bar{x}} S_{m \underline{z}}^{(1-3)} \\
& -2 \sin \theta_{I} \cos (\theta / 2) \sin \varphi \sum_{i, m} b_{i m} I_{i \bar{x}} S_{m \underline{\underline{x}}}^{(1-3)}-\omega_{\mathrm{ef}, S}^{(\mathrm{DQ})} S_{\underline{z}}^{1-3} \\
& +\left[\frac{2}{3} \omega_{Q}+\frac{1}{2}\left(\omega_{e}-\omega_{Q}\right)\right]\left(S_{\underline{\underline{z}}}^{(1-2)}-S_{\underline{\underline{z}}}^{(2-3)}\right),
\end{aligned}
$$

where $\omega_{\mathrm{eff}, S}^{(\mathrm{DQ})}=\left[4 \Delta \Omega_{S}^{2} \cos ^{2}(\theta / 2)+\frac{1}{4}\left(\omega_{e}-\omega_{Q}\right)^{2}\right]^{1 / 2}$. 
This Hamiltonian describes the heteronuclear DQ-coherence transfer in the case of spin-locking (SL) procedure. It is now possible to separate the spin systems involved in the DQ-coherence transfer into two subsystems characterized by the Hamiltonians

$$
\begin{aligned}
& H^{(1)}=-\omega_{\mathrm{eff}, I} I_{\bar{z}}+P_{2}\left(\cos \theta_{I}\right) H_{I I}^{(0)}, \\
& H_{\mathrm{DQ}}^{(2)}=-\omega_{\mathrm{eff}, S}^{(\mathrm{DQ})} S_{\underline{z}}^{(1-3)}
\end{aligned}
$$

and a single-quantum subsystem described by the IIamiltonian

$$
H_{\mathrm{SQ}}^{(2)}=\left[\frac{2}{3} \omega_{Q}+\frac{1}{2}\left(\omega_{e}-\omega_{Q}\right)\right]\left(S_{\underline{z}}^{(1-2)}-S_{\underline{z}}^{(2-3)}\right) .
$$

These IIamiltonians describe the quasi-invariants of motion, i.e. $\left[H^{(1)}, H_{\mathrm{DQ}}^{(2)}\right]=$ $\left[H^{(1)}, H_{\mathrm{SQ}}^{(2)}\right]=\left[H_{\mathrm{DQ}}^{(2)}, H_{\mathrm{SQ}}^{(2)}\right]=0$.

The $I$-spin subsystem described by $H^{(1)}$ Hamiltonian and $S$-spin DQ-subsystem described by $H_{\mathrm{DQ}}^{(2)}$ Hamiltonian are coupled by the perturbation Hamiltonian

$$
\begin{aligned}
H_{\mathrm{p}}= & 2 \cos \theta_{I} \cos (0 / 2) \cos \varphi \sum_{i, m} b_{i m} I_{i \bar{z}} S_{m \bar{z}}^{(1-3)} \\
& +2 \cos \theta_{I} \cos (\theta / 2) \sin \varphi \sum_{i, m} b_{i m} I_{i \bar{z}} S_{m \underline{x}}^{(1-3)} \\
& -2 \sin \theta_{I} \cos (\theta / 2) \sin \varphi \sum_{i, m} b_{i m} I_{i \bar{x}} S_{m \underline{z}}^{(1-3)} \\
& -2 \sin \theta_{I} \cos (\theta / 2) \sin \varphi \sum_{i, m} b_{i m} I_{i \bar{x}} S_{m \underline{x}}^{(1-3)} .
\end{aligned}
$$

The general form of the Hamiltonian, Eq. (29), makes it possible to discuss the adiabatic demagnetization in rotating frame (ADRF) procedure as a limiting case of SL procedure for $I$-spins preparation. If $B_{1 I}$ is changed adiabatically from the value $B_{1 I} \gg B_{\mathrm{L} I}^{\prime}$ to the value $B_{1 I} \ll B_{\mathrm{L} I}^{\prime}$, where $B_{\mathrm{L} I}^{\prime}$ is the local field at the $I$-spins site in their tilted rotating frame [29], the tilt angle $\theta_{I}$ can be considered as approaching the zero value. Thus the Hamiltonians of interest in the ADRF case become from Eqs. (30)

$$
\begin{aligned}
& H^{(1)}=-\left(\Delta \omega_{I}+\Delta \Omega_{I}\right) I_{\bar{z}}+H_{I I}^{(0)}, \\
& H_{\mathrm{DQ}}^{(2)}=-\omega_{\mathrm{eff}, \bar{S}}^{(\mathrm{DQ})} S_{\underline{\underline{Z}}}^{(1-3)}, \\
& H_{\mathrm{SQ}}^{(3)}=\left[\frac{2}{3} \omega_{Q}+\frac{1}{2}\left(\omega_{e}-\omega_{Q}\right)\right]\left(S_{\underline{\underline{z}}}^{(1-2)}-S_{\underline{\underline{z}}}^{(2-3)}\right) .
\end{aligned}
$$

In this case the heteronuclear coupling Hamiltonian has the following form:

$$
\begin{aligned}
H_{\mathrm{p}}= & 2 \cos (\theta / 2) \cos \varphi \sum_{i, m} b_{i m} I_{i \bar{z}} S_{m \underline{z}}^{(1-3)} \\
& +2 \cos (\theta / 2) \sin \varphi \sum_{i, m} b_{i m} I_{i \bar{z}} S_{m \underline{x}}^{(1-3)}
\end{aligned}
$$


The coupling produced by the Hamiltonian $H_{\mathrm{p}}$ will affect only the quasi-invariants of motion $H^{(1)}$ and $H_{\mathrm{DQ}}^{(2)}$ but not the quasi-invariant described by $H_{\mathrm{SQ}}^{(2)}$ Ilamiltonian.

The above Hamiltonians describe the process of DQ-heteronuclear coherence transfer for all regions of the sample if the gradient strength $G_{z}$ satisfies the condition $\gamma_{s} G_{z} d_{z} \ll \omega_{Q}$, where $d_{z}$ is the sample dimension. This condition assure that the $S$-irradiation RF-field frequency remains for any position close to the Larmor frequency $\omega_{0 . S}$. If this condition is not satisfied the single-quantum coherences are also produced.

\section{Spatial localized spin dynamics}

We want to discuss in the following the spin dynamics for a heteronuclear DQ-coherence transfer via dipolar order. The basic experimental scheme is presented in Fig. 1. The abundant $I=1 / 2$ spins are prepared in a state of low spin temperature by adiabatic demagnetization in the rotating frame (ADRF) procedure in the absence of any field gradient. The contact pulse is applied at the Larmor frequency of $S$-quadrupole spins in the presence of a main magnetic field gradient.

The spin systems which participate in a double-quantum heteronuclear coherence transfer can be described by the formal inverse spin temperatures defined as [30]

and

$$
\beta^{(1)}(t)=\frac{\operatorname{Tr}\left\{H^{(1)} \rho_{\mathrm{DQ}}(t)\right\}}{\operatorname{Tr}\left\{\left(H^{(1)}\right)^{2}\right\}}
$$

$$
\beta^{(2)}(t)=\frac{\operatorname{Tr}\left\{H_{\mathrm{DQ}}^{(2)} \rho_{\mathrm{DQ}}(t)\right\}}{\operatorname{Tr}\left\{\left(H^{(1)}\right)^{2}\right\}},
$$

where $\rho_{\mathrm{DQ}}$ is the density operator in the same quantum mechanical representation as the DQ-Hamiltonian given by Eq. (29).

The time evolution of the inverse spin temperature as a function of the experimental preparation conditions and physical characteristics of the subsystem can be obtained from the corresponding Liouville-von Neuman equation of the whole system. In the (i) limit of: fast-dipolar fluctuation correlation time $\tau_{c}$, i.e. $\tau_{\mathrm{c}} \ll T_{I S}^{(\mathrm{DQ})}$, where $T_{I S}^{(\mathrm{DQ})}$ is the heteronuclear DQ-coherence transfer time and (ii) second order in the coúpling Hamiltonian, the following equation is valid [30]:

$$
\frac{\partial \beta^{(2)}(t)}{\partial t}=\frac{\beta^{(1)}(t)-\beta^{(2)}(t)}{\cdot T_{I S}^{(\mathrm{DQ})}}
$$

where

$$
\begin{aligned}
\left(T_{I S}^{(\mathrm{DQ})}\right)^{-1} & =-\frac{1}{\operatorname{Tr}\left\{\left(H_{\mathrm{DQ}}^{(2)}\right)^{2}\right\}} \int_{0}^{\infty} \mathrm{d} \tau \operatorname{Tr}\left\{\left(\widehat{H}_{\mathrm{p}} H_{\mathrm{DQ}}^{(2)}\right)\right. \\
& \left.\times \exp \left(-\mathrm{i}\left(\hat{H}^{(1)}+\widehat{H}_{\mathrm{DQ}}^{(2)}\right) \tau\right)\left(\widehat{H}_{\mathrm{p}} H_{\mathrm{DQ}}^{(2)}\right)\right\} .
\end{aligned}
$$

From Eqs. (30), (31) and (36) we can write for the ADRF-case

$$
\left(T_{I S}^{(\mathrm{DQ})}(z)\right)^{-1}=4 \cos ^{2}(\theta / 2) \sin ^{2} \varphi M_{2}^{I S} J_{z}\left(\omega_{\mathrm{eff}, S}{ }^{(\mathrm{DQ})}\right) \text {. }
$$


The above expression describes the spatially localized heteronuclear coherence transfer rate. The angle $\varphi$, the effective frequency $\omega_{\mathrm{eff}, S}^{(\mathrm{DQ})}$ and $M_{2}^{I S}-$ the van Vleck second moment of the magnetic resonance line determined by the cross-coupling dipolar interaction depend on the $z$-coordinate of the slice. This dependence is not written explicitly in Eq. (37) in order to simplify the equations.

The $z$-slice spectral density functions which describe the dipolar fluctuations in the "thermal bath" represented by the $I$-spin system is given by

$$
J_{z}(\omega)=\int_{0}^{\infty} \mathrm{d} \tau \cos \omega \tau C_{z}(\tau)
$$

which is the Fourier transforms of the localized dipolar fluctuation autocorrelation functions [30],

$$
\begin{aligned}
C_{z}(\tau)= & \operatorname{Tr}\left\{\left(\sum_{i} b_{i m} I_{i z}\right) \exp \left[-\mathrm{i} P_{2}\left(\cos \theta_{I}\right) H_{I I}^{(0)} \tau\right]\right. \\
& \left.\times\left(\sum_{i} b_{i m} I_{i z}\right)\right\} / \operatorname{Tr}\left\{\left(\sum_{i} b_{i m} I_{i z}\right)^{2}\right\} .
\end{aligned}
$$

In the above equations $I_{i}$ represents the $I$-spin operator for the $i$-spin inside of the $z$-slice.

We can define the normalized DQ-relaxation ratio as

$$
r^{\mathrm{DQ}}=\left(T_{I S}^{(\mathrm{DQ})}(z)\right)^{-1} /\left(T_{I S}^{(\mathrm{DQ})}(0)\right)^{-1} \text {. }
$$

From Eqs. (37) and (40) we have

$$
r_{\mathrm{ADRF}}^{\mathrm{DQ}}=\frac{\sin ^{2} \varphi(z)}{\sin ^{2} \varphi(0)} \frac{J_{z}\left(\omega_{\mathrm{eff}, S}^{(\mathrm{DQ})}(z)\right)}{J_{z}\left(\omega_{\mathrm{eff}, S}^{(\mathrm{DQ})}(0)\right.} .
$$

Equation (41) is written for the case of a homogeneous sample, i.e., $M_{2}^{I S}(z)=$ $M_{2}^{I S}(0)$ for any $z$-slice. For the case of on-resonance irradiation we have $\theta_{I}(0)=$ $\varphi(0)=\pi / 2$.

In order to analyze the spatial dependence of the relaxation rate we have to evaluate the dipolar spectral density function. A detailed computation of this function is very complicated [30]. To simplify the evaluation we will consider that the dipolar fluctuation autocorrelation function could be described in a good approximation by a Lorentzian function, i.e.

$$
C_{z}(\tau) \cong \frac{1}{1+\tau^{2} / \tau_{c}^{2}}
$$

The correlation time is $\tau_{\mathrm{c}}=\left(2 / M_{2}\right)^{1 / 2}$, where $M_{2}$ is the second moment of the corresponding spectral density function which can be expressed as a function of lattice sums. An estimation of this correlation time is $\tau_{\mathrm{c}} \approx 2 \pi / \omega_{\mathrm{L} I}^{\prime}$.

With these assumptions Eq. (41) can be written as

$$
r_{\mathrm{ADRF}}^{\mathrm{DQ}}=\sin ^{2} \varphi(z) \exp \left\{-\tau_{\mathrm{c}}\left[\omega_{\mathrm{eff}, S}^{(\mathrm{DQ})}(z)-\omega_{\mathrm{eff}, S}^{(\mathrm{DQ})}(0)\right]\right\} \text {. }
$$

We can remark from the above equations that the DQ-heteronuclear coherence transfer rate has a strong dependence on the position in the sample. This 
spatial dependence is mainly determined by the $z$-dependence of the dipolar spectral density function.

In order to analyze the spatial localization of the normalized relaxation rates we will consider the particular case of benzene- $d_{1}$, for which the DQ-heteronuclear coherence transfer between $I={ }^{1} \mathrm{H}$ and $S={ }^{2} \mathrm{D}$ has been carefully experimentally examined [10].

The deuterium quadrupole interaction frequency of monodeutero-benzene is $2 \nu_{Q}=70.4 \mathrm{kHz}$ and the maximum of its powder NMR spectrum is at $\nu_{Q}=$ $\pm 35.2 \mathrm{kIIz}$. The local field strength $\omega_{L I}$ in frequency units of the proton was calculated [10] from the linewidth of the proton spectrum of benzene- $d_{1}$ and has the value $\omega_{L I}^{\prime} / 2 \pi \cong 2.75 \mathrm{kIz}$. Using this value we can estimate $\tau_{\mathrm{c}}=2 \pi / \omega_{\mathrm{L} I}^{\prime}=364 \mu \mathrm{s}$.

The spatial dependence of $r_{\mathrm{ADRF}}^{\mathrm{DQ}}$ normalized coherence transfer rates as a function of field gradient strength, quadrupole interaction frequencies and $S$-radiofrequency field strength is presented in Fig. 2. It is evident from Fig. 2a that the localization effect is relatively high for moderate value of gradient strength.

The sensitivity of relaxation rate profiles to the value of quadrupole interaction frequency is presented in Fig. 2b. A small dependence of the linewidth profiles as a function of $\nu_{Q}$ is evidenced. In the ADRF case the spatial localization decreases for higher values of $\nu_{1 S}$ (cf. Fig. 3c). We intend to analyze now the spatial dependence of the ratio between DQ- and SQ-coherence transfer rates. For SQ-heteronuclear coherence transfer we will consider the spin $S=1$ with no quadrupole interactions, i.e. $\nu_{Q}=0$. From the above equations and the expressions [30] for $r^{\mathrm{SQ}}$ we can write

$$
r_{\mathrm{ADRF}}^{\mathrm{DQ} / \mathrm{SQ}}=\frac{4 \cos ^{2}(\theta / 2) \sin ^{2} \varphi(z)}{\sin ^{2} \theta_{S}(z)} \exp \left\{-\tau_{\mathrm{c}}\left[\omega_{\mathrm{eff}, S}^{(\mathrm{DQ})}(z)-\omega_{\mathrm{eff}, S}^{(\mathrm{SQ})}(z)\right]\right\},
$$

where $\tan \theta_{S}(z)=\omega_{1 S} /\left(\gamma_{S} G_{z} z\right)$ for $\Delta \omega_{S}=0$ and $\omega_{\mathrm{ef}, S}^{(\mathrm{SQ})}(z)=\left[\omega_{1 S}^{2}+\left(\gamma_{S} G_{z} z\right)^{2}\right]^{1 / 2}$.

In Fig. 3 we have represented the spatial dependence of $r_{\mathrm{ADRF}}^{\mathrm{DQ} / \mathrm{SQ}}$ for different values of $\nu_{1}$. In the central region of the sample the $r_{\mathrm{ADRF}}^{\mathrm{DQ}}$ exceeds with almost five magnitude order the $r_{\mathrm{ADRF}}^{\mathrm{SQ}}$-rate. This is related to the reduction of $\nu_{1 S}$ with the factor $\nu_{1 S} / \nu_{Q}$ for DQ-coherence transfer process and much faster cross-relaxation rate as a result of a much better match with the $I$-spin local frequency.

At the time $t$ from the beginning of the contact pulse after the quasi-equilibrium state for quasi-invariants of motion was reached the local DQ-density operator has the following form

$$
\rho_{\mathrm{DQ}}(z, t) \cong \frac{1}{\operatorname{Tr}\{1\}}\left[1-\beta_{I}(z, t) H_{I I}^{(0)}(z)-\beta_{S}^{(\mathrm{DQ})}(z, t) H_{\mathrm{DQ}}^{(2)}(z)\right],
$$

where $\operatorname{Tr}\{1\}=(2 S+1)^{N_{S}}(2 I+1)^{N_{I}}$ with $N_{S}$ and $N_{I}$ the number of the spins $S$ and $I$ from the $z$-slice.

As a result of preparation procedure and coherence transfer we have $\beta_{I}(z, t)$ and $\beta_{S}^{\mathrm{DQ}}(z, t) \gg \beta_{\mathrm{L}}\left(\beta_{\mathrm{L}}=1 / k T_{\mathrm{L}}\right.$, where $T_{\mathrm{L}}$ is the lattice temperature $)$.

The time evolution of inverse effective spin temperature for the two quasi-invariants coupled in the process of heteronuclear coherence transfer can be described 

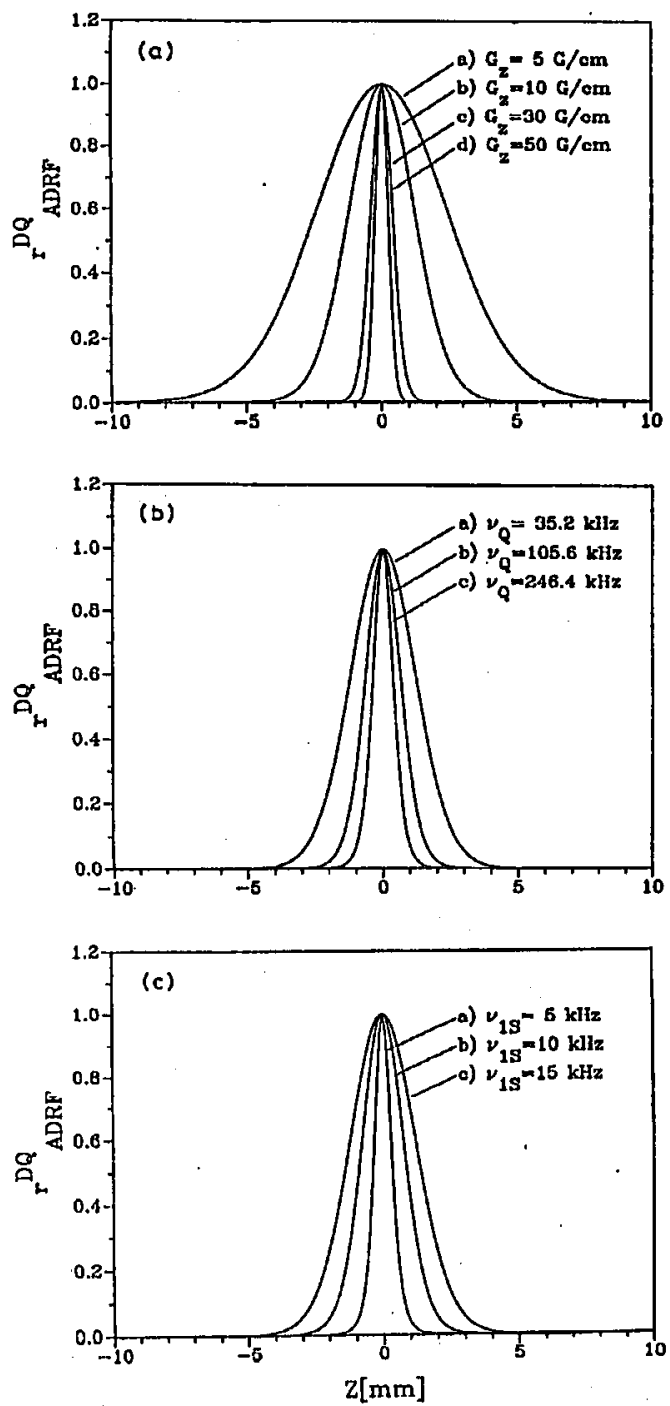

Fig. 2. The spatial dependence of DQ-heteronuclear coherence transfer rates $r_{\mathrm{ADRF}}^{\mathrm{DQ}}$. In all these cases $\nu_{Q}=35.2 \mathrm{kHz}$. (a) The $r_{\mathrm{ADRF}}^{\mathrm{DQ}}$ spatial profile is represented as a function of $G_{z}$ for $\nu_{1 S}=15 \mathrm{kHz}$. (b) The dependence of the spatial profiles as a function of $\nu_{Q}$ is represented for $\nu_{1 S}=15 \mathrm{kHz}$ and $G_{z}=10 \mathrm{G} / \mathrm{cm}$. The dependence of the spatial profiles for $G_{z}=10 \mathrm{G} / \mathrm{cm}$ as a function of $\nu_{1 S}$ is presented in (c).

by the following coupled differential equations [31]:

$$
\frac{\partial \beta_{S}^{\mathrm{DQ}}(z, t)}{\partial t}=-\frac{1}{T_{I S}^{\mathrm{DQ}}}\left[\beta_{S}^{(\mathrm{DQ})}(z, t)-\beta_{I}(z, t)\right]
$$




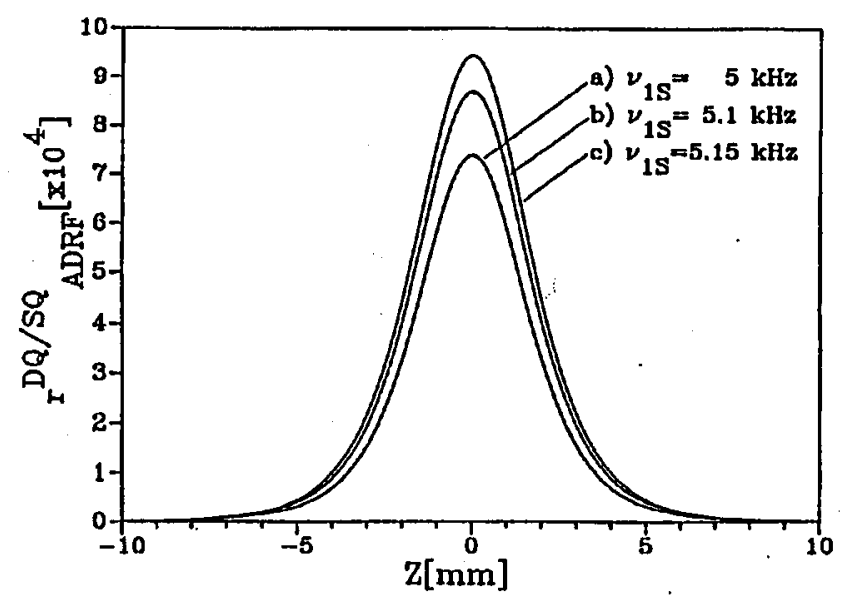

Fig. 3. The spatial dependence of the $r_{\mathrm{ADRF}}^{\mathrm{DQ} / \mathrm{SQ}}$ ratio. The ratio represented in the figure versus $z$-coordinate was evaluated for $G_{z}=2 \mathrm{G} / \mathrm{cm}$ and $\nu_{Q}=35.2 \mathrm{kHz}$.

and

$$
\frac{\partial \beta_{I}(z, t)}{\partial t}=-\frac{\varepsilon^{(\mathrm{DQ})}(z)}{T_{I S}^{\mathrm{DQ}}}\left[\beta_{I}(z, t)-\beta_{S}^{(\mathrm{DQ})}(z, t)\right],
$$

where any spin-lattice relaxation process for $I$ and $S$ spins has been ignored. The range of time $t$, for which Eqs. (46) are valid, is $t>T_{2 I}^{*}$ (to avoid the transient oscillation regime), where $T_{2 I}^{*}$ is the effective dephasing time of $I$ spins transverse magnetization. The spatial localization ratio $\varepsilon^{(\mathrm{DQ})}(z)$ between heat capacities of the quasi-invariants of motion is given by

$$
\varepsilon_{\mathrm{ADRF}}^{(\mathrm{DQ})}(z)=\frac{N_{S}(z)\left[\omega_{\mathrm{ef}, S}^{(\mathrm{DQ})}(z)\right]^{2}}{N_{I}(z)\left[\omega_{\mathrm{L} I}^{\prime}(z)\right]^{2}} .
$$

The initial conditions of Eqs. (46) are related to the saturation pulse sequence applied at the Larmor frequency of $S$ spins for which $\beta_{S}^{(\mathrm{DQ})}(z, 0)=0$ as to the preparation of $I$-spins.

For ADRF-procedure performed in the absence of a field gradient the pros: duced low spin temperature is spatially distributed as a result of different values for $\omega_{\mathrm{L} I}^{\prime}$ in the case of inhomogeneous sample, i.e.

$$
\beta_{I}(z, 0)=\beta_{\mathrm{L}} \omega_{0 I} / \omega_{\mathrm{L} I}^{\prime}(z) \text {. }
$$

The solutions of Eqs. (46) with the above initial conditions are

$$
\beta_{S}^{(\mathrm{DQ})}(z, t)=\beta_{I}(z, 0) \frac{1}{1+\varepsilon^{(\mathrm{DQ})}(z)}\left\{1-\exp \left[-\left(1+\varepsilon^{(\mathrm{DQ})}(z)\right) t / T_{I S}^{(\mathrm{DQ})}(z)\right]\right\}
$$

and

$$
\beta_{I}(z, t)=\beta_{I}(z, 0) \frac{1}{1+\varepsilon^{(\mathrm{DQ})}(z)}\left\{1+\varepsilon^{(\mathrm{DQ})} \exp \left[-\left(1+\varepsilon^{(\mathrm{DQ})}(z)\right) t / T_{I S}^{(\mathrm{DQ})}(z)\right]\right\}
$$


The formal inverse spin-temperature, $\beta_{S}^{(\mathrm{DQ})}(z, t)$, measures the degree of which the double-quantum coherence is created by heteronuclear transfer from $I=1 / 2$ spins. The process of DQ-heteronuclear coherence transfer is spatially localized and the coefficient $\beta_{S}^{(\mathrm{DQ})}(z, t)$ describes this spatial distribution.

\section{Heteronuclear transferred double-quantum coherence by indirect detection}

The indirect detection procedure which will be analyzed in the following is presented in Fig. 4. We will discuss here only the case of DQ-coherence excitation and detection via dipolar order which is a much more universal technique [11] as compared with the spin-locking procedure especially for systems with large quadrupolar splitting. The duration of the contact pulse for excitation of DQ-coherence is denoted by $t_{c}$ and for the contact detection pulse by $t$. The dipolar order at the end of excitation DQ-coherence period is eliminated by a "magic" $54.7^{\circ}$-pulse [32]. The created dipolar order at the end of detection period is transferred by ADRF-procedure [31] into the Zeeman order by a $(\pi / 2)_{-x}$ pulse along the static magnetic field. The spatial distributed Zeeman order is read by a solid-echo in the presence of a read magnetic field gradient.
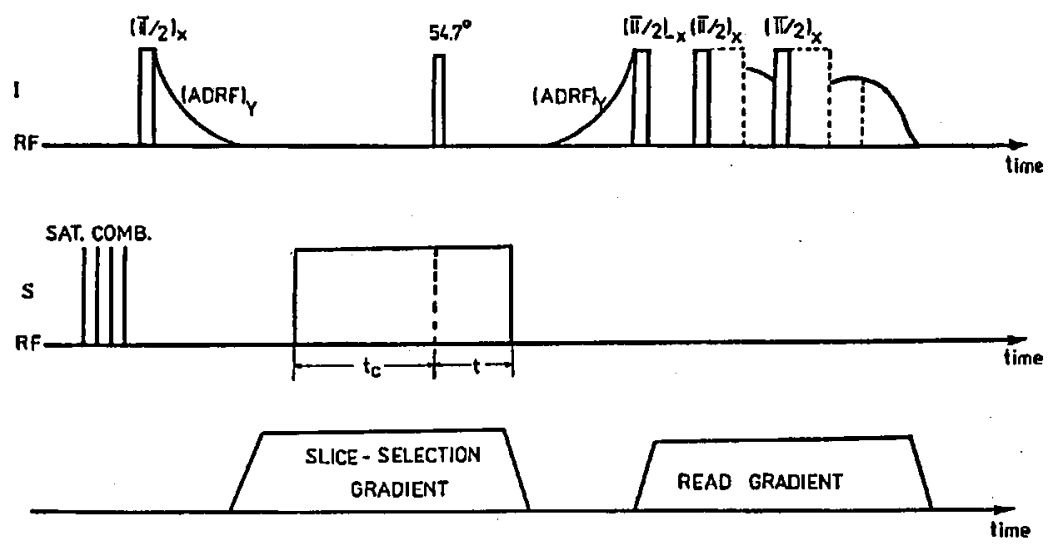

Fig. 4. The cyclic pulse sequence used for recording the spatial distribution of heteronuclear transferred DQ-coherence via dipolar order. The produced DQ-coherence during the time interval $t_{c}$ is transferred back during $t$ interval into $I$-spin SQ-coherence using dipolar order, stored Zeeman order by the pulse $(\pi / 2)_{-x}$ and finally the read solid echo pulse sequence acting in the presence of a read field gradient.

At the end of the coherence transfer contact pulse of length $t_{c}$ the inverse effective spin temperature are given by Eqs. (49) with $t=t_{\mathrm{c}}$ and $\varepsilon_{\mathrm{ADRF}}^{(\mathrm{DQ})}$. With these initial conditions the system of coupled differential equations (46) can be solved and the solution for inverse spin temperature of $I$-spin system at the $t$ 
moment of time during the back coherence transfer is given by

$$
\begin{aligned}
& \beta_{I}\left(z, t+t_{\mathrm{c}}\right)=\beta_{\mathrm{L}} \frac{\omega_{0 I}}{\omega_{\mathrm{L} I}^{\prime}(z)} \frac{\varepsilon_{\mathrm{ADRF}}^{(\mathrm{DQ})}(z)}{1+\varepsilon_{\mathrm{ADRF}}^{(\mathrm{DQ})}(z)}\left[1-\exp \left\{-\frac{\left[1+\varepsilon_{\mathrm{ADRF}}^{(\mathrm{DQ})}(z)\right] t_{\mathrm{c}}}{T_{I S}^{(\mathrm{DQ})}(z)}\right\}\right] \\
& \times\left\{1-\frac{1}{1+\varepsilon_{\mathrm{ADRF}}^{(\mathrm{DQ})}(z)}\left[\varepsilon_{\mathrm{ADRF}}^{(\mathrm{DQ})}(z)+\exp \left(-\frac{\left[1-\varepsilon_{\mathrm{ADRF}}^{(\mathrm{DQ})}(z)\right] t}{T_{I S}^{(\mathrm{DQ})}(z)}\right)\right]\right\} .
\end{aligned}
$$

We are interested to observe the SQ-coherence of $I$-spin system. The relevant density operator in the rotating reference frame is given by

$$
\rho_{R}\left(z, t+t_{\mathrm{c}}\right)=\frac{1}{\operatorname{Tr}\{1\}}\left[1-\beta_{I}\left(t+t_{\mathrm{c}}\right) H_{I I}^{(0)}(z)\right] .
$$

The inverse local spin temperature at the end of ADRF process $\beta_{I f}(z)$ can be evaluated [29] considering the process to be isentropic. It is given by

$$
\beta_{I f}(z)=\beta_{I}\left(z, t+t_{\mathrm{c}}\right) \frac{\omega_{\mathrm{LI}}^{\prime}(z)}{\omega_{1 I}} .
$$

The process of adiabatic remagnetization is performed in the absence of the field gradient. In spite of that, the $I$-spin temperature is spatially localized as a result of previous history of the sample, restricted spin-diffusion [16] and sample heterogeneities.

The final local density operator at the end of ADRF process is

$$
\rho_{f R}(z)=\frac{1}{\operatorname{Tr}\{1\}}\left\{1+\beta_{I f}(z) \omega_{1 I} I_{y}(z)\right\} .
$$

If we consider an ideal read pulse sequence (generally a solid-echo pulse sequence) the SQ $I$-spin coherence profile can be evaluated from, the above equations

$$
\left\langle I_{y}(z)\right\rangle=\operatorname{Tr}\left\{I_{y}(z) \rho_{f R}(z)\right\}=\frac{N_{I}(z)}{4} \beta_{I}\left(z, t+t_{\mathrm{c}}\right) \omega_{\mathbf{L} I}^{\prime}(z) .
$$

The recorded spatial profiles reflect the spatial localization process of direct and inverse DQ-coherence transfer, which is evident from the expression of $\beta_{I}\left(z, t+t_{c}\right)$.

The normalized DQ-coherence transfer profile detected via $I$-spin signal is

$$
S_{\mathrm{ADRF}}^{(I)}(z)=\frac{\left\langle I_{y}(z)\right\rangle}{\left\langle I_{y}(0)\right\rangle} \text {. }
$$

For a homogeneous sample (i.e. $N_{I}(z)=N_{I}(0)$ and $\omega_{\mathrm{L} I}(z)=\omega_{\mathrm{LI}}(0)$ ) Eq. (54) can be rewritten as

$$
S_{\mathrm{ADRF}}^{(I)}(z)=\frac{\beta_{I}\left(z, t+t_{\mathrm{c}}\right)}{\beta_{I}\left(0, t+t_{\mathrm{c}}\right)} .
$$

The spatial distribution of the cyclic DQ-heteronuclear transfer described by Eq. (56) was represented in Fig. 5 for $t=t_{c}$ and different values of gradient strength, total contact pulse duration and the $S$-radiofrequency field amplitude. The slice profiles are determined by a "convolution" between the spatial distribution of the direct and the inverse DQ-coherence transfer. The width of the slice profiles shows a strong dependence on gradient strength $G_{z}$. For a gradient strength 

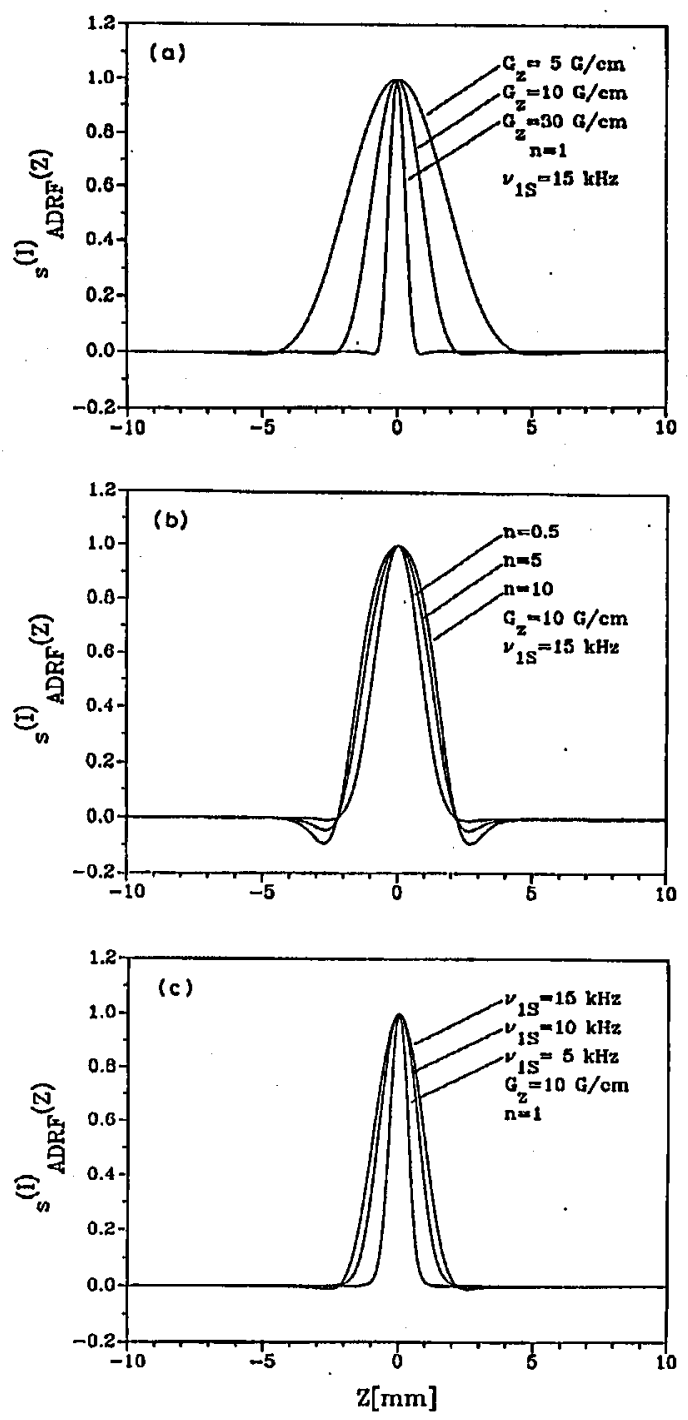

Fig. 5. The spatial profiles of DQ-coherence produced by cyclic heteronuclear transfer via dipolar order using indirect detection for $t=t_{\mathrm{c}}$. The case of ${ }^{1} \mathrm{H}-{ }^{2} \mathrm{H}$ in benzene- $\mathrm{d}_{1}$ was considered. The spatial profiles as a function of gradient strength, contact pulse duration $t=n T_{I S}^{(\mathrm{DQ})}(0)$ and $\nu_{1 S}$ frequency were represented in (a), (b) and (c), respectively.

of $G_{z}=30 \mathrm{G} / \mathrm{cm}$ the localization is in the order of $500 \mu \mathrm{m}$ (cf. Fig. 5a). The width does not depend essentially on duration of contact time $n=t / T_{I S}^{(\mathrm{DQ})}(0)$, in the investigated range (cf. Fig. $5 \mathrm{~b}$ ), but is sensitive to the strength of the contact pulse $\nu_{1 s}$. The localization becomes better for a smaller value of $\nu_{1 s}$ (cf. Fig. $5 \mathrm{c}$ ). 


\section{Conclusions}

The cross-polarization of rare spins from dipolar order state of abundant spins is often advantageous, because a much smaller radio-frequency contact pulse applied to $S$-spins suffices. Since in the case of quadrupole $S$-spins radiofrequency fields are scaled by $\omega_{1 S} / \omega_{Q}$ this advantage becomes severe in DQ-coherence transfer. The high-resolution spectra of quadrupole nuclei (e.g. $\left.{ }^{2} \mathrm{II},{ }^{14} \mathrm{~N},{ }^{23} \mathrm{Na},{ }^{27} \mathrm{Al}\right)$ in solid can be recorded from DQ-coherence giving access to important structural and dynamic information. The indirect detection via protons will increase the sensitivity of the technique and will extend the accessible range of quadrupole interactions.

The process of heteronuclear DQ-coherence transfer from $I=1 / 2$ spins to $S=1$ spins in the presence of a field gradient is strongly spatially localized. Depending on the gradient strength, contact pulse duration and amplitude the slice profile has a quasi-triangular shape. Moreover, the effect of the field gradient on space localization is amplified by the coherence order and by cyclic transfer.

The dependence of the linewidth at the half intensity of the DQ excited profiles on gradient strength follows the general dependence law, $\Delta z_{1 / 2} G_{z}=$ const, where the value of the constant depends on the experimental conditions and the sample internal parameters. We estimate that for moderate field gradient strength of about $100 \mathrm{G} / \mathrm{cm}$ a slice width of the order of $10 \mu \mathrm{m}$ can be excited.

The slice selection produced simultaneously with the DQ-coherence will open the possibility to design compact, high-efficient pulse sequences for volume localized spectroscopy and imaging of quadrupole nuclei in high magnetic field.

\section{Acknowledgments}

We thank Prof. Dr. Rainer Kimmich and Dr. Siegfried IIafner for valuable discussions.

\section{References}

[1] R. Kimmich, D.E. Demco, S. Hafner, in: Magnetic Resonance Microscopy, Eds.

B. Blümich, W. Kuhn, VCH, Weinheim 1992.

[2] P. Blümler, B. Blümich, in: NMR Basic Principles and Progress, Eds. P. Diehl, E. Fluck, R. Kosfeld, Vol. 30, Springer, Berlin 1994.

[3] S. Hafner, D.E. Demco, R. Kimmich, Solid State Nucl. Magn. Reson., in press.

[4] N.M. Szeverenyi, G.E. Maciel, J. Magn. Reson. 60, 460 (1984).

[5] C.G. Fry, A.C. Lind, M.F. Davies, D.W. Duff, G.E. Maciel, J. Magn. Reson. 83, 656 (1989).

[6] D.G. Cory, W.S. Veeman, J. Phys. E 22, 180 (1989).

[7] J.B. Miller, A.N. Garroway, J. Magn. Reson. 85, 255 (1989).

[8] E. Günter, B. Blümich, H.W. Spiess, Macromolecules 25, 3315 (1992).

[9] P. Brunner, M. Reinold, R.R. Ernst, J. Chem. Phys. 73, 1086 (1980).

[10] S. Vega, T.W. Shattuch, A. Pines, Phys. Rev. A 22, 638 (1980).

[11] M. Reinhold, P. Brunner, R.R. Ernst, J. Chem. Phys. 74, 184 (1981).

[12] T.K. Pratum, M.P. Klein, J. Magn. Reson. 55, 421 (1983). 
[13] C. Fernandez, J.P. Amoureux, Chem. Phys. Lett. 242, 449 (1995).

[14] E. Rommel, S. Hafner, R. Kimmich, J. Magn. Reson. 86, 264 (1990).

[15] S. Hafner, E. Rommel, R. Kimmich, J. Magn. Reson. 88, 449 (1990).

[16] D.E. Demco, R. Kimmich, S. Hafner, H.W. Weber, J. Magn. Reson. 94, 317 (1991).

[17] D.G. Cory, J.B. Miller, A.N. Garroway, J. Magn. Reson. 90, 544 (1990).

[18] R.A. Wind, C.S. Yannoni, J. Magn. Reson. 36, 269 (1990).

[19] S. Matsui, J. Magn. Reson. 97, 335 (1992).

[20] D.E. Demco, R. Kimmich, in: Extended Abstracts, Eds. A. Anagostrupoulos, F. Milia, A. Simopoulos, 26th Congress Ampere, Athens 1992.

[21] S. Hafner, D.E. Demco, R. Kimmich, Chem. Phys. Lett. 187, 53 (1991).

[22] D.E. Demco, I. Ardelean, Acta Phys. Pol. A 86, 407 (1994).

[23] D.E. Demco, S. Hafner, I. Ardelean, R. Kimmich, Appl. Magn. Reson. 9, 491 (1995).

[24] C. Kunze, R. Kimmich, D.E. Demco, J. Magn. Reson. A 101, 277 (1993).

[25] D.E. Demco, H. Köster, R. Kimmich, J. Magn. Reson. A 110, 136 (1994).

[26] II. Köster, R. Kimmich, J. Magn. Reson. B 102, 177 (1993).

[27] A. Wokaun, R.R. Ernst, J. Chem. Phys. 67, 1752 (1977).

[28] S. Vega, J. Chem. Phys. 68, 5518 (1978).

[29] M. Goldman, Spin Temperature and Nuclear Magnetic Resonance in Solids, Oxford, U.P., London 1970.

[30] D.E. Demco, J. Tegenfeldt, J. Waılgh, Phys. Rev. B 11, 4133 (1975).

[31] M. Mehring, Principles of High Resolution NMR in Solids, Springer-Verlag, Berlin 1983.

[32] S. Emid, J. Magn. Reson. 42, 147 (1981). 